\title{
Genome-wide analysis of HSP70 gene superfamily in Pyropia yezoensis (Bangiales, Rhodophyta): identification, characterization and expression profiles in response to dehydration stress
}

Xinzi Yu ${ }^{1,2}$, Zhaolan Mo ${ }^{1,2}$, Xianghai Tang ${ }^{1,2}$, Tian Gao ${ }^{1,2}$ and Yunxiang Mao ${ }^{3,4^{*}}$

\begin{abstract}
Background: Heat shock proteins (HSPs) perform a fundamental role in protecting plants against abiotic stresses. Individual family members have been analyzed in previous studies, but there has not yet been a comprehensive analysis of the HSP70 gene family in Pyropia yezoensis.

Results: We investigated 15 putative HSP70 genes in Py. yezoensis. These genes were classified into two sub-families, denoted as DnaK and Hsp1 10. In each sub-family, there was relative conservation of the gene structure and motif. Synteny-based analysis indicated that seven and three PyyHSP70 genes were orthologous to HSP70 genes in Pyropia haitanensis and Porphyra umbilicalis, respectively. Most PyyHSP70s showed up-regulated expression under different degrees of dehydration stress. PyyHSP70-1 and PyyHSP70-3 were expressed in higher degrees compared with other PyyHSP70s in dehydration treatments, and then expression degrees somewhat decreased in rehydration treatment. Subcellular localization showed PyyHSP70-1-GFP and PyyHSP70-3-GFP were in the cytoplasm and nucleus/cytoplasm, respectively. Similar expression patterns of paired orthologs in $P$ y. yezoensis and Py. haitanensis suggest important roles for HSP70s in intertidal environmental adaptation during evolution.
\end{abstract}

Conclusions: These findings provide insight into the evolution and modification of the PyyHSP7O gene family and will help to determine the functions of the HSP70 genes in Py. yezoensis growth and development.

Keywords: Pyropia yezoensis, HSP70 gene family, Genome-wide analysis, Dehydration stress, Phylogeny, Gene structure, Expression pattern, Subcellular localization

\section{Background}

Heat shock proteins (HSPs) are found in almost all organisms, from bacteria to humans [1]. In plants, members of the family of HSPs act in cell protection through the

\footnotetext{
*Correspondence: yxmao@hntou.edu.cn

${ }^{3}$ Key Laboratory of Utilization and Conservation of Tropical Marine Bioresource (Hainan Tropical Ocean University), Ministry of Education, Sanya 572022, China

Full list of author information is available at the end of the article
}

folding and translocation of nascent proteins and the refolding of denatured proteins under both stress and non-stress conditions [2,3]. HSPs can be divided into five families based on molecular weight: HSP70, 70-kDa heat shock protein; HSP90 and HSP100 family; HSP60, chaperonin family; and sHSP, small heat shock protein. Of these, HSP70 is widely conserved and has been shown to play roles in development and defense mechanisms under various stresses.

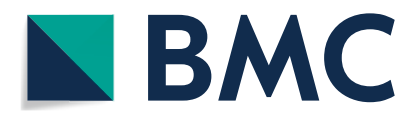

(c) The Author(s) 2021. Open Access This article is licensed under a Creative Commons Attribution 4.0 International License, which permits use, sharing, adaptation, distribution and reproduction in any medium or format, as long as you give appropriate credit to the original author(s) and the source, provide a link to the Creative Commons licence, and indicate if changes were made. The images or other third party material in this article are included in the article's Creative Commons licence, unless indicated otherwise in a credit line to the material. If material is not included in the article's Creative Commons licence and your intended use is not permitted by statutory regulation or exceeds the permitted use, you will need to obtain permission directly from the copyright holder. To view a copy of this licence, visit http://creativecommons.org/licenses/by/4.0/. The Creative Commons Public Domain Dedication waiver (http://creativeco mmons.org/publicdomain/zero/1.0/) applies to the data made available in this article, unless otherwise stated in a credit line to the data. 
The HSP70 gene family contains three highly conserved domains: a C-terminal domain about $10 \mathrm{kDa}$ in size that can bind substrate, an intermediate domain $15 \mathrm{kDa}$ in size, and an $\mathrm{N}$-terminal domain (NBD) $44 \mathrm{kDa}$ in size that binds ATP [4]. Plant HSP70 genes have been localized to four locations: the cell nucleus/cytoplasm, endoplasmic reticulum (ER), plastids, and mitochondria, with different functions in different locations [5, 6]. Deficiency of some cytosolic HSP70s led to severe growth retardation, and heat treatment of plants deficient in HSP7O genes dramatically increases mortality, indicating that cytosolic HSP70s plays an essential role during normal growth and in the heat response by promoting the proper folding of cytosolic proteins $[7,8]$.

Ectopic expression of a cytosolic CaHSP70-2 gene resulted in altered expression of stress-related genes and increased thermotolerance in transgenic Arabidopsis [9]. Cytosolic HSP70A in Chlamydomonas regulates the stability of cytoplasmic microtubules [10, 11]. Transgenic tobacco plants that over-expressed nuclear-localized NtHSP70-1 exhibited decreased fragmentation and degradation of nuclear DNA during heat-/drought-stress [6, 12]. Knockout experiments indicate that the import of stromal HSP70s into the chloroplast stroma is essential for plant development and important for the thermotolerance of germinating seeds [13]. Transgenic tobacco plants constitutively expressing elevated levels of BIP (an ER-localized HSP70 homologue) exhibited tolerance to water deficit by preventing endogenous oxidative stress [14]. In rice, the BIP1/OsBIP3 gene, encoding HSP70 in the ER, regulates the stability of XA21 protein to interfere with XA21-mediated immunity [15]. Mitochondrial HSP70 can suppress programmed cell death in rice protoplasts by maintaining mitochondrial membrane potential and inhibiting the amplification of reactive oxygen species (ROS) [16]. However, the biological functions of most HSP70s in nori have not yet been elucidated, partly due to a lack of information about coding genes or other genomic information. Pyropia yezoensis (Bangiales, Rhodophyta) is an economically important seaweed that is cultivated in the intertidal zones of China coastlines [17]. The production and quality of the cultivated $P y$. yezoensis thalli are significantly influenced by intertidal environmental stress. Tidal exposure imposes considerable environmental stress on intertidal seaweeds due to altered irradiance levels [18], temperature changes [19], and direct effects from desiccation [20,21].

In this study, all of the non-redundant members of HSP70 genes in Py. yezoensis were screened from available, high-quality, chromosomal-level genomes. We determined the characteristics of PyyHSP7O genes based on the physicochemical properties, genomic locations, and conserved motifs, promoters, and analyzed the phylogenetic relationships of these genes. In addition, the expression levels of the PyyHSP7O genes were analyzed under dehydration and rehydration conditions. Finally, highly expressed PyyHSP70 proteins were localized in Arabidopsis protoplasts. Our findings will be useful resources for future studies of the functions of HSP7O genes in algae, which will help us understand the evolution of HSP70 genes in different species.

\section{Results \\ Genome-wide identification of PyyHSP7O genes in Py. yezoensis}

After verification, the sequence information was obtained from the Py. yezoensis genome for 15 putative PyyHSP70s. The basic information of PyyHSP70 genes (including genomic position, gene length, intron number, amino acid number, isoelectric point (pI), molecular weight, CDS, subcellular localization, and instability index) is listed in Table 1. The predicted PyyHSP70 protein sequences ranged from 276 amino acids to 934 amino acids, and the molecular weights ranged from 29.59 to $96.07 \mathrm{kDa}$. Analysis with the Expasy online tool revealed instability index values of PyyHSP70s that ranged from 21.76 to 48.25 , with a single PyyHSP70 member (PyyHSP70-8) having an instability index greater than 40, indicating an unstable protein. Of the 15 PyyHSP70 proteins, 11 members are predicted to localize to the nucleus/cytoplasm, one to the ER, one to the mitochondria, and two to the chloroplasts. The genes either had no introns or one intron, with eight and seven members respectively (Fig. 1A). The 15 members of PyyHSP70 were distributed on all three chromosomes, with an uneven distribution in the genome. Chromosome 1 had the highest density of PyyHSP7O genes, nine members (Fig. 1B).

\section{Conserved motifs and phylogenetic analysis of PyyHSP70s}

To better understand the structural characteristics of PyyHSP70 proteins, a multiple sequence alignment was performed of the HSP70 domains of all 15 PyyHSP70 proteins and the EcDNAK protein, as shown in Figure S1. The two functional domains (ATPase domain and peptide-binding domain) were present in all PyyHSP70s. The ATPase domains of PyyHSP70-6, PyyHSP70-7, and PyyHSP70-15 were shorter, and lacked three signature motifs that are characteristic of the ATPase domain of HSP70 family members (Table S1). Additionally, the peptide-binding domain of PyyHSP70-2 was shorter, and much shorter $\mathrm{C}$-terminal sub-domains were present in PyyHSP70-2 and PyyHSP70-7 (Table S1).

Twelve consensus motifs were found in PyyHSP70 proteins using the MEME motif search tool (Fig. 2, Table S2). Motifs 1, 2, 5, 6, 7, 9, 10, 11, and 12 were identified in the 


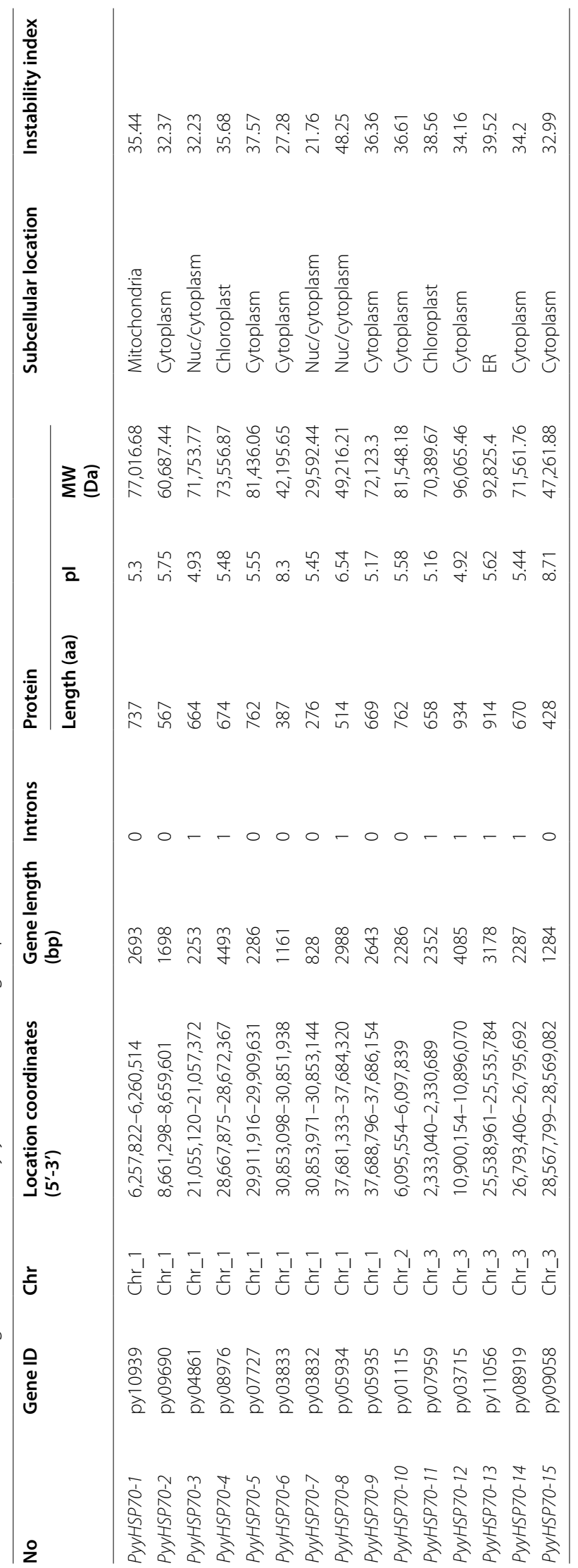


A

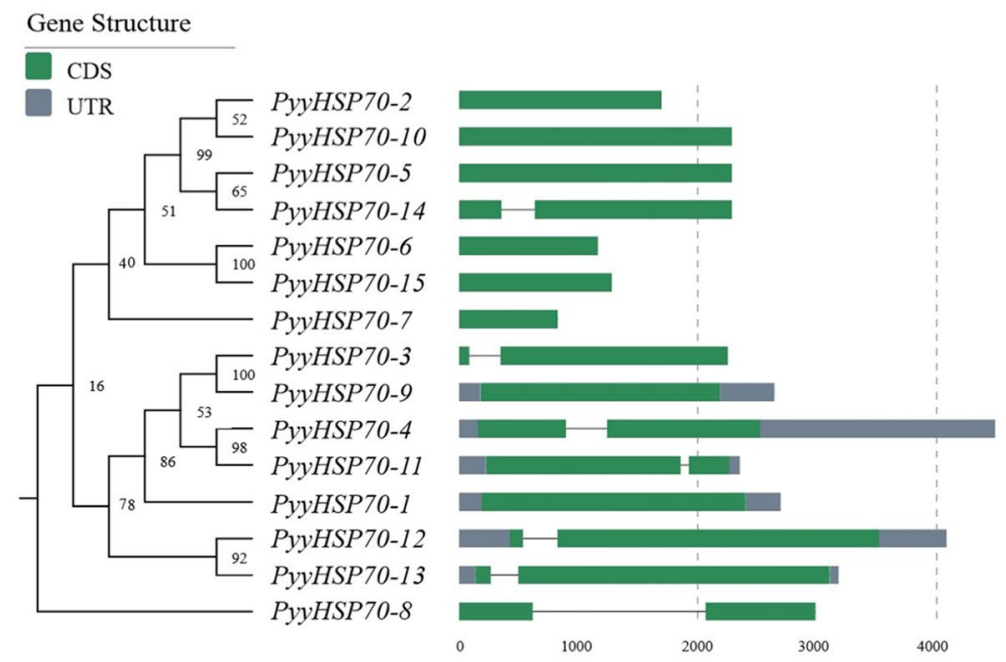

B
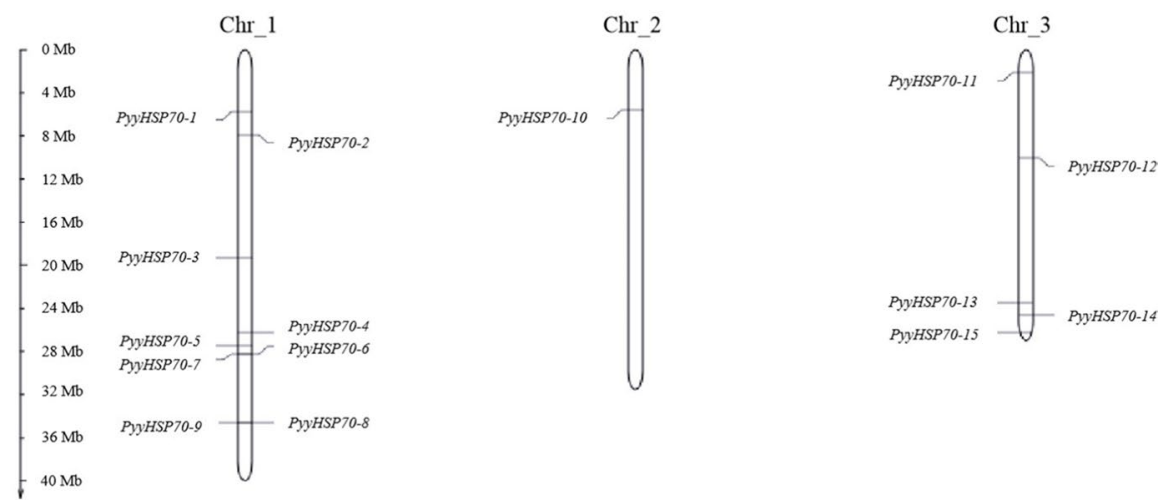

Fig.1 A Phylogenetic tree and gene structures of PyyHSP70 genes. B Chromosomal location of PyyHSP70 genes

ATPase domain, and motifs 3,4 , and 8 were identified in the peptide-binding domain. Only motifs $3,4,6$, and 7 were detected in all PyyHSP70 members of the DnaK subfamily, and only motif 2 was detected in all PyyHSP70 members of the Hsp110 subfamily.

An unrooted phylogenetic tree was constructed to visualize the evolutionary relationships between HSP70 members, using 76 HSP70 protein sequences from nine species (Table S3). As shown in Fig. 2, these HSP70s were classified into two subfamilies (the DnaK subfamily and the Hsp110 subfamily). The DnaK subfamily was further divided into four groups based on localization (cytoplasm, ER, mitochondria, and plastid). The HSP70 proteins from different species were more closely related to those in the same subfamily than to others in the same species. For example, cytosolic PyyHSP70-4 was more closely to PyhHSP70-5 than PyyHSP70-11.

For the PyyHSP7O family members, orthologs from Py. yezoensis and Py. haitanensis (seven pairs) or Porphyra umbilicalis (three pairs) were identified, indicating there may have been common ancestral genes of the HSP7O family before differentiation of the three species (Fig. 3). In addition, a subclade of six genes (PyyHSP70-2, PyyHSP70-5, PyyHSP70-6, PyyHSP70-10, PyyHSP70-14 and PyyHSP70-15) in the cytoplasm group implied the proximity of these sequences and potential paralogous relationships by duplication events after the divergence of the two Pyropia species. The $\mathrm{Ka} / \mathrm{Ks}$ ratios of the parolog pairs in the subclade were calculated and the results ranged from $0.6327-1.3487$ (Table 2). The $\mathrm{Ka} / \mathrm{Ks}$ ratios for five pairs were less than but close to one, indicating slightly negative selection; the other two pairs' $\mathrm{Ka} / \mathrm{Ks}$ ratios were greater than one, suggesting positive selection. All seven orthologous events of Pyropia exhibited $\mathrm{Ka} / \mathrm{Ks}$ ratios far less than 1 (Table S4). 

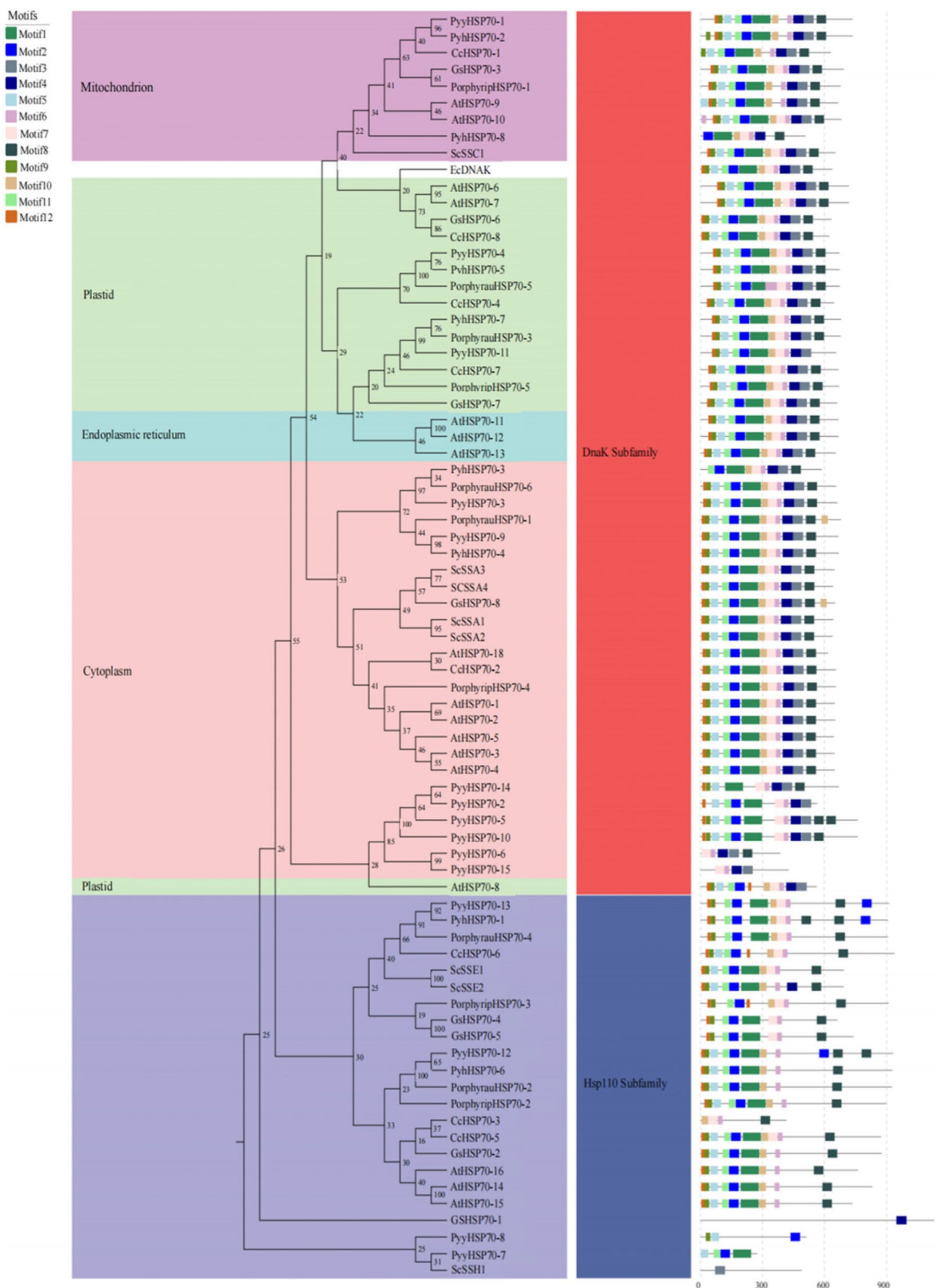

Fig. 2 Phylogenetic (left) and conserved motif (right) analysis of HSP70 proteins. Motif analysis was performed using the online MEME program. Different colors of boxes represent different motifs in the corresponding positions of each protein 


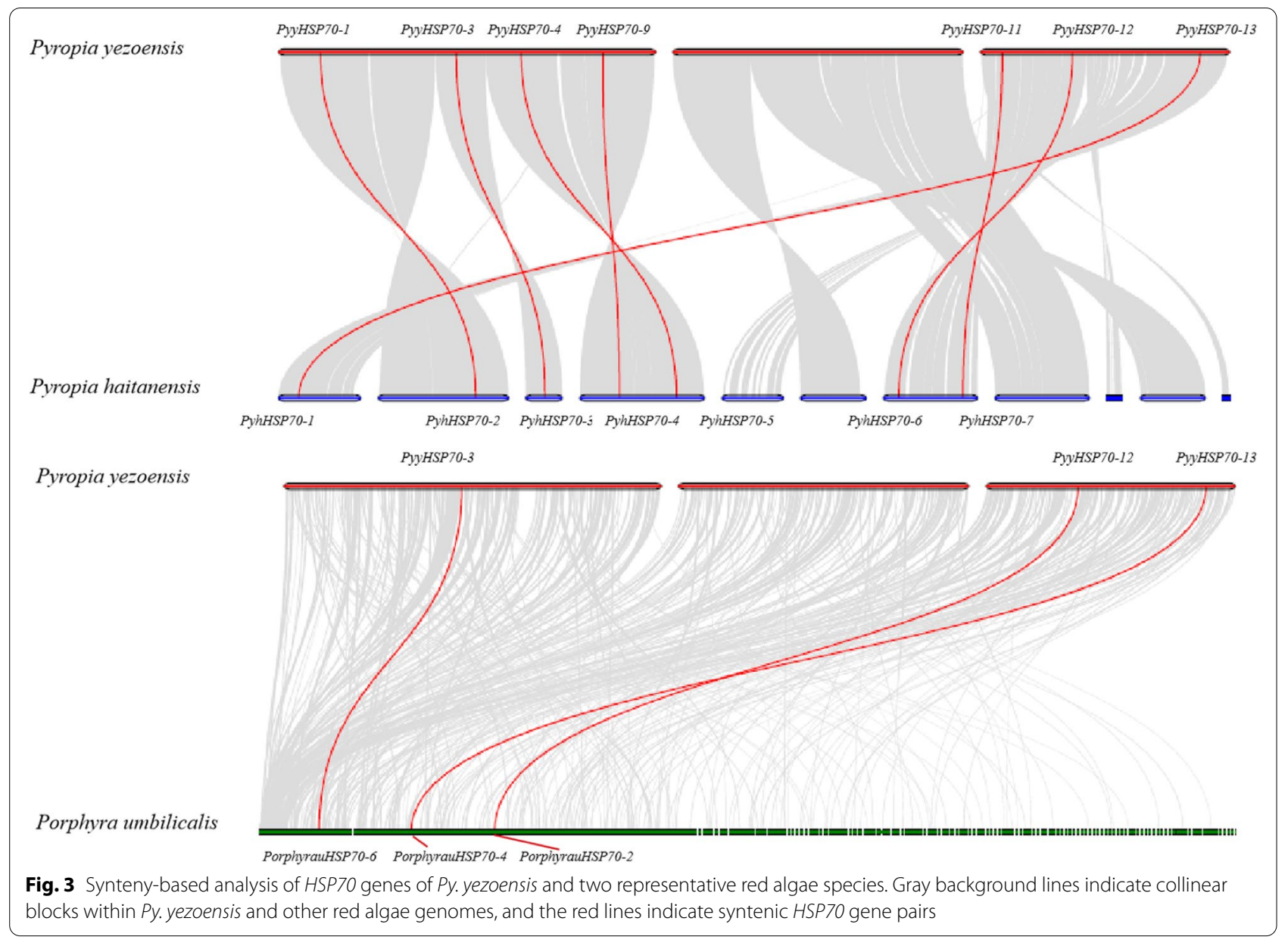

\section{Cis-regulatory element analysis of the PyyHSP7O gene family}

The regulatory roles of the identified PyyHSP70 genes were further studied by analysis of the 2000 bp region upstream of these genes. We searched the promoter sequences using the PlantCARE tool for seven regulatory elements previously found to be involved in various stresses: ABRE, CGTCA-motif, TGACG-motif, TCAelement, MYB-binding sites (MBS), LTR, and DRE (Table 3). Fourteen of these genes possessed several

Table $2 \mathrm{Ka}$, Ks, and $\mathrm{Ka} / \mathrm{Ks}$ values for duplicated paralog pairs in Py. yezoensis

\begin{tabular}{|c|c|c|c|c|}
\hline Seq 1 & Seq 2 & $\mathrm{Ka}$ & Ks & $\mathrm{Ka} / \mathrm{Ks}$ \\
\hline PуyHSP7-2 & PуyHSP7-10 & 1.0282 & 0.9313 & 1.1040 \\
\hline PуyHSP7-5 & PyyHSP7-10 & 0.0284 & 0.0448 & 0.6327 \\
\hline PyyHSP7-5 & РуyHSP7-2 & 0.9768 & 1.0797 & 0.9048 \\
\hline PyyHSP7-5 & PуyHSP7-14 & 0.9766 & 1.0596 & 0.9217 \\
\hline РуyHSP7-6 & PуyHSP7-15 & 0.9664 & 1.1183 & 0.8642 \\
\hline PyyHSP7-14 & РуyHSP7-2 & 0.9569 & 1.1361 & 0.8423 \\
\hline PyyHSP7-14 & PyyHSP7-10 & 1.0968 & 0.8133 & 1.3487 \\
\hline
\end{tabular}

ABRE elements (all but PyyHSP70-4); all PyyHSP70 genes contained the CGTCA-motif and MYB-binding sites (MBS); 13 of 15 PyyHSP70 genes contain TGACGmotifs (all but PyyHSP70-13 and PyyHSP70-15); 12 of 15 PyyHSP7O genes contain CRE/DRE elements (all except PyyHSP70-2, PyyHSP70-4, and PyyHSP70-13). Besides, we also searched three types of heat shock elements (HSEs), perfect type (nTTCnnGAAnnTTCn), gap type (nTTCnnGAAnnnnnnnTTCn) and step (S) type (nTTCnnnnnnnTTCnnnnnnnTTCn) [22], in these promoter sequences. Only one S-type HSE was detected in PyyHSP70-3. The detection of these abiotic response elements suggests that the PyyHSP7O genes may be extensively involved in stress responses, thereby increasing the range of mechanisms that organisms could emply to escape or better cope with adverse environmental effects.

\section{Expression patterns of PyyHSP70 genes under dehydration treatments}

To further clarify the potential ability of the PyyHSP7O genes to respond to dehydration stress, RNA-Seq data 
Table 3 Summary of stress-inducible cis-elements in the promoter regions of PyyHSP70 genes

\begin{tabular}{|c|c|c|c|c|c|c|c|}
\hline No & $\mathrm{ABRE}^{\mathrm{a}}$ & CGTCA-motif ${ }^{b}$ & TGACG-motif ${ }^{c}$ & TCA-element ${ }^{d}$ & $\mathrm{MBS}^{\mathrm{e}}$ & LTR $^{f}$ & $\mathrm{DRE}^{\mathrm{g}}$ \\
\hline РууНSP70-1 & 4 & 6 & 5 & 0 & 1 & 0 & 2 \\
\hline РууНSP70-2 & 9 & 2 & 5 & 0 & 6 & 0 & 0 \\
\hline РуyНSP70-3 & 3 & 3 & 2 & 0 & 1 & 1 & 1 \\
\hline РууНSP70-4 & 0 & 3 & 2 & 0 & 9 & 0 & 0 \\
\hline PуyHSP70-5 & 11 & 3 & 6 & 0 & 6 & 0 & 1 \\
\hline РуyHSP70-6 & 4 & 2 & 7 & 0 & 3 & 0 & 3 \\
\hline PуyHSP70-7 & 1 & 1 & 6 & 1 & 3 & 0 & 2 \\
\hline РуyНSP70-8 & 6 & 4 & 3 & 0 & 5 & 0 & 4 \\
\hline PуyHSP70-9 & 10 & 3 & 2 & 0 & 4 & 1 & 5 \\
\hline PyyHSP70-10 & 9 & 4 & 6 & 0 & 4 & 0 & 1 \\
\hline PуyHSP70-11 & 2 & 3 & 4 & 0 & 5 & 0 & 2 \\
\hline PyyHSP70-12 & 8 & 1 & 5 & 0 & 3 & 0 & 4 \\
\hline PуyHSP70-13 & 5 & 2 & 0 & 0 & 2 & 0 & 0 \\
\hline PуyHSP70-14 & 9 & 7 & 5 & 0 & 9 & 1 & 1 \\
\hline PуyHSP70-15 & 5 & 2 & 0 & 0 & 3 & 0 & 1 \\
\hline
\end{tabular}

a cis-acting element involved in abscisic acid response

b cis-acting regulatory element involved in MeJA-response

c cis-acting regulatory element involved in the MeJA-response

d cis-acting element involved in salicylic acid response

e MYB binding site involved in drought-inducibility

f cis-acting element involved in low-temperature response

g cis-acting element involved in dehydration, low-temperature, and salt stresses

were analyzed. Expression analysis of PyyHSP7O under dehydration stress revealed low $(<0.3)$ or no expression from seven genes in all treatments, but the other eight PyyHSP70 genes exhibited higher expression (Fig. 4). The expression of PyyHSP70-1 and PyyHSP70-3 gradually increased with increased dehydration stress, and the expression level slightly decreased with subsequent rehydration treatment. The expression levels of PyyHSP70-11 increased with increased water loss and continued to increase during rehydration. The expression levels of PyyHSP70-8, PyyHSP70-4, and PyyHSP70-13 first increased and then decreased as the degree of dehydration deepened. The expression levels of PyyHSP70-8 and PyyHSP70-13 increased during rehydration, and PyyHSP70-4 experienced an increase of expression for one dehydration condition (AWC20) and then decreased during rehydration. These RNA-seq expression patterns were verified by detecting the expression patterns of the PyyHSP7O genes by qRT-PCR (Fig. 5). The measured expression levels of most genes were highly consistent with the levels determined by RNA-seq, except for PyyHSP70-13.

\section{The subcellular localization of PyyHSP70-1} and PyyHSP70-3 proteins

PyyHSP70-1 and PyyHSP70-3 showed the biggest expression changes in response to dehydration stress, so we next determined the subcellular localization of these two proteins. PyyHSP70-1 was localized to the cytoplasm, and PyyHSP70-3 localized to the nucleus/cytoplasm (Fig. 6), basically consistent with the predicted results (Table 1, Fig. 6).

\section{Disscussion}

Daily changes in tide height cause air exposure to seaweed, triggering rapidly-changing physical stresses such as dehydration, high temperature, and different irradiance levels [23]. Because they live in the challenging habitat of the intertidal zone, intertidal macroalgae have adapted a set of protective mechanisms to survive [24]. Some intertidal seaweeds are highly tolerant to desiccation. Species of the genera Pyropia and Porphyra (Bangiales, Rhodophyta) inhabit the upper intertidal zone and can lose up to $95 \%$ of cellular water content during maximum low tide [18]. HSP70 is a superfamily of molecular chaperones widely distributed in eukaryotic cells. These proteins play important roles under abiotic stress by participating in many protein folding processes. However, the HSP70 superfamily of Py. yezoensis was not 


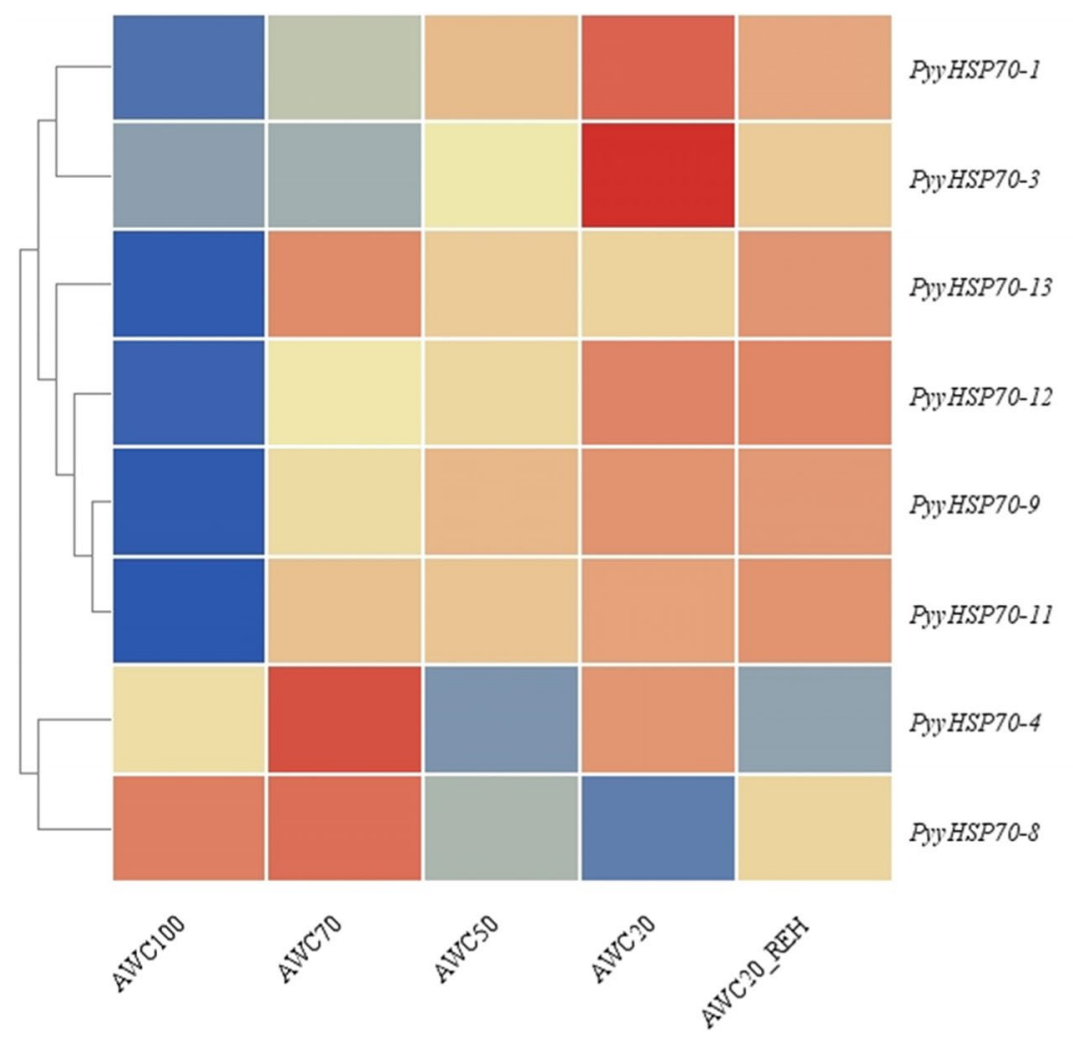

Fig. 4 Heatmap of the expression patterns of PyyHSP70 genes under dehydration and rehydration treatments: absolute water content 100\% (AWC100, control), absolute water content 70\% (AWC70), absolute water content 50\% (AWC50), absolute water content 20\% (AWC20), rehydrated 30 min after 20\% of water loss (AWC20_30min). The color bar (right) represents $\log _{2}$ expression levels (FPKM). The tree (left) represents clustering result of PyyHSP70s' expression patterns

previously characterized. In this work, we comprehensively analyzed the characteristics, expression patterns under dehydration stress, and the subcellular localization of PyyHSP70s.

\section{Evolution analysis of $H S P 70$ genes}

In this study, we identified 15 HSP70 domain-containing genes in the $P$. yezoensis genome that constitute the HSP70 superfamily, including 11 DnaK subfamily genes and $4 \mathrm{Hsp} 110$ subfamily genes. We also analyzed the genomes of five other red algae and identified 36 HSP7O genes. We found no direct relationship between genome size and the number of HSP70 genes in red algae. For example, we identified eight HSP7O genes in Py. haitanensis (genome size: $53 \mathrm{Mb}$ ), eight genes in Galdieria sulphuraria (genome size: $14 \mathrm{Mb}$ ), and eight genes in Chondrus crispus (genome size: $105 \mathrm{Mb}$ ). This diversity in the number of red algae $H S P 70$ genes indicated that the $H S P 70$ gene family has utilized different evolutionary strategies in different species.

PyyHSP70s proteins were divided into two sub-families, similar to those reported by previous analysis of
HSP70s in A. thaliana and yeast [5, 25]. The DnaK subfamily was further divided into four groups based on localization. The number of HSP7O genes from the six red algae was basically same in each group of the DnaK subfamily, except the Cytoplasm group which contained more members of the PyyHSP7O gene family due to paralogous duplication events. Paralogous duplication events were not evident in the other five red algae, further implying that PyyHSP70s expanded according to species-specific approaches during evolution. We found no expression for these paralogous PyyHSP70 genes with intact gene structures in dehydration treatments, and also no expression of these genes was detected in response to other abiotic/biotic stresses of P.yezoensis [20, 21, 26]. HSP genes in other species were previously identified that also did not appear to be expressed under tested conditions, but the reason has not yet been determined [27, 28]. Interesting, two-pairs of PyyHSP7O paralogs showed positive selection, suggesting new functions that should be verified by further experiments. 


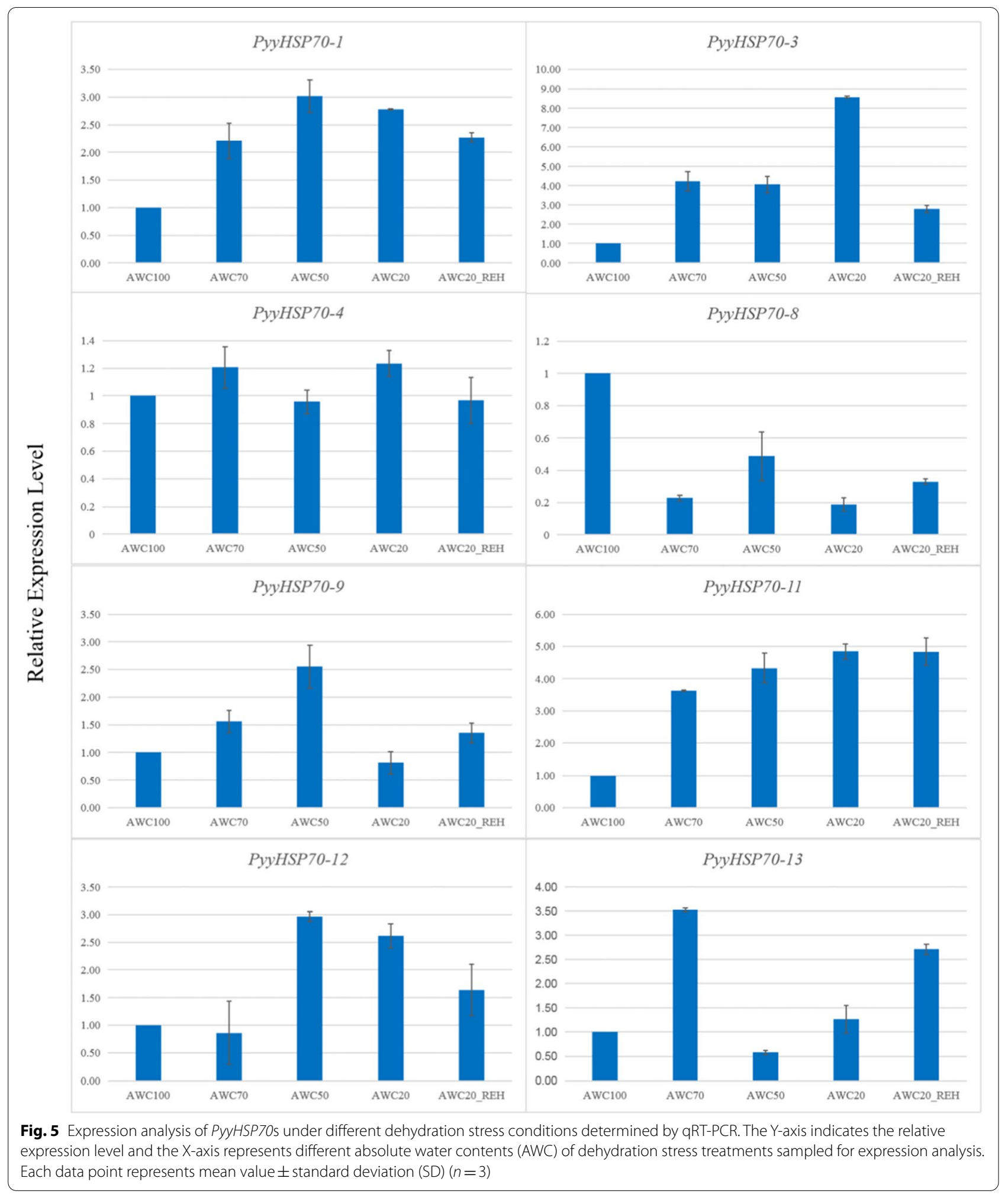

HSP70 genes play essential roles in response to dehydration stress

Previous studies have found abundant HSEs in the promoter regions of $H S P 70$ genes that become active in response to heat shock and other temperature treatments in higher plants $[2,29]$. However, we found few HSE and 


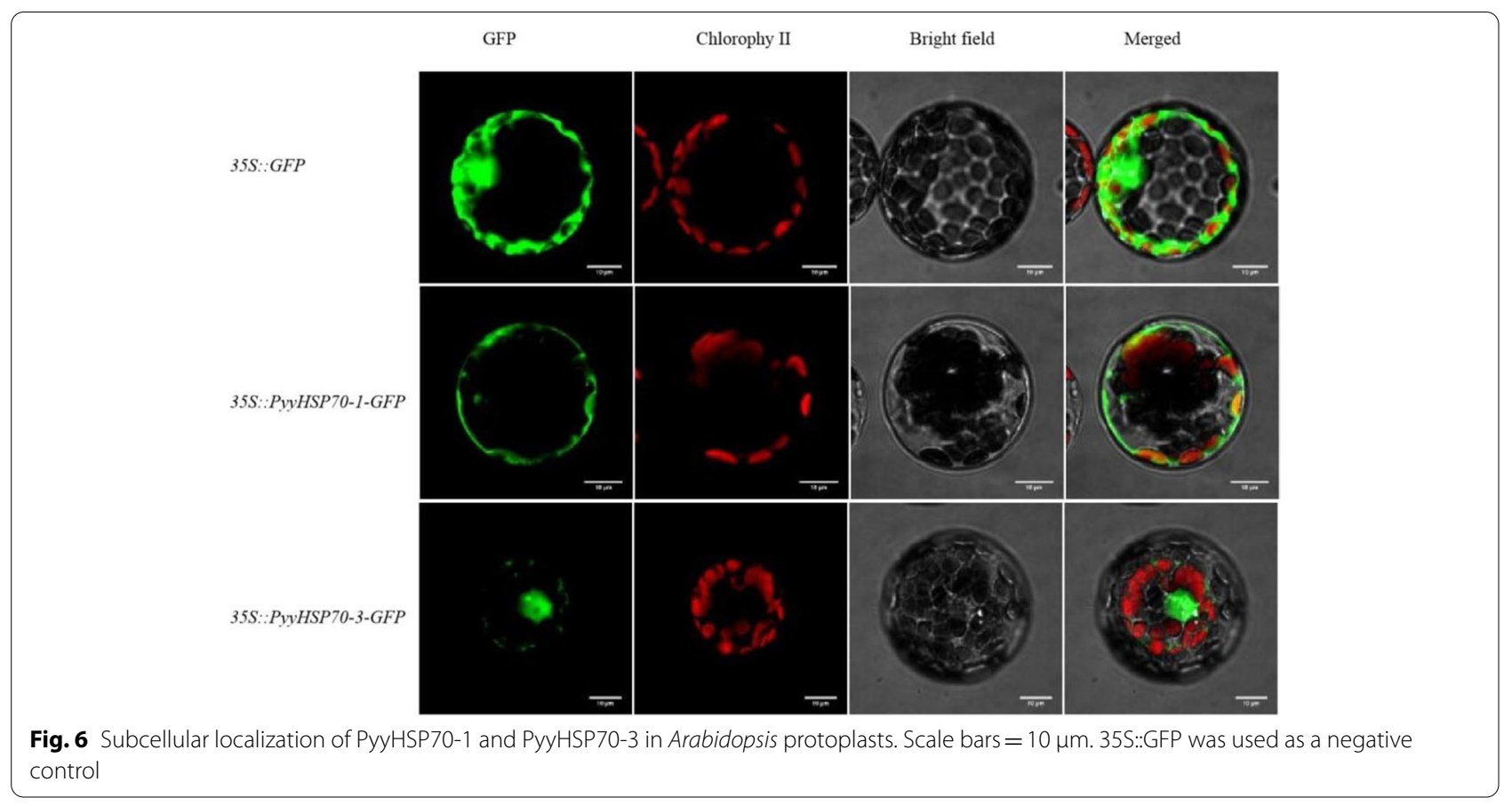

LTR in the promoter regions of PyyHsp70 genes. Py. yezoensis live on intertidal rocks, where they experience repeated cycles of dehydration and rehydration. Cisregulatory element analysis showed that most PyyHSP7O gene promoter sequences contained cis-elements associated with dehydration stress. For example, the ABRE motif conserved in drought response genes [30, 31], MYB binding sites (MBS) involved in drought-inducibility, and CRT/DRE elements associated with dehydration and salt stresses [32, 33]. The results suggest that PyyHSP70s might be significantly related to dehydration response.

Intermittent desiccation stress caused by tidal changes is a significant abiotic factor affecting intertidal seaweed species. This stress can affect the physiology of organisms, mainly through oxidative stress causing destabilization of proteins, leading to loss of membrane integrity [34-36]. Desiccation results in increased expression of tolerance genes, such as genes encoding HSPs and related transcriptional factors [37, 38]. These mechanisms may also function in intertidal seaweed to tolerate desiccation. Several HSP70s have also been found to help protect against desiccation damage by assisting protein-folding processes involved in stress and affecting the proteolytic degradation of unstable proteins [39]. HSP70s have also received attention in marine organisms as a kind of biomarker of stress, because their expression is highly variable in the presence or absence of stimuli [40-42]. Zhou et al. (2011) suggested that analysis of HSP70 genes could be utilized to evaluate algae tolerance to stresses and monitor coastal environmental changes [43].
Tang et al. (2016) found that moss plants overexpressing PpcpHSP70-2 highly induced by dehydration treatment showed dehydration tolerance [44]. We found that more than half of PyyHSP7O genes exhibited increased transcription levels with increasing degree of dehydration. We also found significantly increased expression of some PyyHSP70 genes, especially PyyHSP70-1 and PyyHSP70-3, upon reaching a water content of $20 \%$, with down-regulated expression after rehydration. This finding was consistent with that of a previous study that showed that HSP70s played important roles only in the response to extreme desiccation stress [41].

Like Py. yezoensis, Py.haitanensis also lives in the intertidal zone and experiences repeated dehydration and rehydration (though at a different temperature). These two species are evolutionarily very close, both belonging to Pyropia. Paired orthologs between Py.yezoensis and Py. haitanensis showed strong purifying selection and similar trends in expression (Table S4, Figure S2), suggesting that these $H S P 70$ orthologs play important roles in dehydration treatments of laver. Therefore, it is important to study the HSP70 genes involved in the dehydrationinduced response of $P y$. yezoensis to further explain the stress resistance and environmental adaptation of intertidal algae.

\section{Conclusions}

The Py. yezoensis genome contains 15 members of the HSP7O gene family, and these genes are unevenly distributed on three chromosomes. The gene structures 
and phylogenetic analysis suggest a complex evolution history of this gene family in Py. yezoensis. The analysis reveals that the PyyHSP7O family has experienced gene duplication events after species divergence relative to other red algae. Most HSP70s showed up-regulated expression under different degrees of dehydration stress, especially PyyHSP70-1 and PyyHSP70-3 which showed much higher expression levels in dehydration treatments and slightly decreased expression after rehydration treatment. Similar expression trends of orthologs of Py.yezoensis and Py.haitanensis in dehydration treatments demonstrate the important roles of these proteins in intertidal environmental adaptation during evolution. PyyHSP70-1-GFP and PyyHSP70-3-GFP were localized in the cytoplasm and the nucleus/cytoplasm, respectively. This overview of this gene family should facilitate further studies of the HSP70 gene family, particularly in regards to their evolutionary history and biological functions.

\section{Methods \\ Genome-wide identification of HSP70 proteins in Py. yezoensis}

The Py. yezoensis genome and protein sequences were deposited to DDBJ/ENA/GenBank as accession WMLA00000000 [17]. To identify candidate Py. yezoensis HSP70 protein sequences, the Hidden Markov model (HMM) profile of the HSP70 domain was downloaded from the Pfam (http://www.sanger.ac.uk/Software/ Pfam/) database (Pfam:PF00012) and then submitted as a query in a HMMER (e-value $<1 \mathrm{e}^{-5}$ ) search (https:// www.ebi.ac.uk/Tools/hmmer/) of the Py. yezoensis protein database. The obtained protein sequences were screened and verified for the presence of the HSP70 domain using SMART (http://smart.embl-heidelberg. de/) tools [45], CDD (http://www.ncbi.nlm.nih.gov/Struc ture/cdd/wrpsb.cgi) and InterProScan (http://www.ebi. ac.uk/interpro/result/InterProScan/). The same process was used to obtain the other four red algae HSP70 family genes from their genome databases [46-49]. For the PyyHSP70 genes, we determined the chromosomal locations, genomic sequences, full coding sequences, protein sequences, and the sequence of the 2000 nucleotides upstream of the translation initiation codon. The molecular weight $(\mathrm{Da})$ and isoelectric point $(\mathrm{pI})$ was calculated for each gene using the Compute $\mathrm{pI} / \mathrm{Mw}$ tool from ExPASy (http://www.expasy.org/tools/) [50]. The subcellular localization of proteins was determined by analysis of the WoLF PSORT, Predotar, PSORT, SherLoc2, CELLO, and Softberry databases, and decided based on consensus localization for two or more algorithms. Schematic images of the chromosomal locations of the PyyHSP70 genes were generated using MapGene2Chrom software (http://mg2c.iask.in/mg2c_v2.1/), according to the chromosomal position information in the NCBI database.

\section{Gene structure analysis and identification of conserved motifs}

To investigate the diversity and structure of members of the PyyHSP7O gene family, we compared the exon/ intron organization of the cDNA sequences and the corresponding genomic DNA sequences of $H S P 70$ using EVOLVIEW (https://evolgenius.info//evolview-v2/). In addition, the amino acid sequences were subjected to "predict the domain and motif analyses" online with MEME (http://meme-suite.org) [51]. The parameters were as follows: number of repetitions, any; maximum number of motifs, 12; and optimum motif widths, 2 to 300 amino acid residues.

\section{Multiple alignment and phylogenetic analysis}

We constructed two phylogenetic trees, one with only PyyHSP70 protein sequences and the other including 76 HSP70 protein sequences from different species. The gene and protein sequences of Arabidopsis thaliana, Escherichia coli and yeast were acquired from previous studies [2, 25, 52, 53] and accession GCA_008690995.1 (NCBI). Multiple sequence alignment to full predicted HSP70 protein sequences was performed with Muscle in Molecular Evolutionary Genetics Analysis (MEGA) 7.0 software using default parameters [54]. Sequence alignments were performed with ClustalX software [55]. Phylogenetic trees were constructed using MEGA7.0 with the Neighbor-Joining (NJ) method, and a bootstrap analysis was conducted using 1000 replicates with pairwise gap deletion mode.

\section{Gene duplication and $\mathrm{Ka} / \mathrm{Ks}$ analysis}

The microsynteny between Py. yezoensis, Py. haitanensis, and Po. umbilicalis was analyzed by MCScanX with the default parameters [56]. The criteria used to analyze potential gene duplications included: (1) the length of the sequence alignment covered $\geq 70 \%$ of the longer gene; and (2) the similarity of the aligned gene regions $\geq 70 \%$ [57]. Non-synonymous (Ka) substitution and synonymous (Ks) substitution were calculated for each duplicated PyyHsp70 gene using KaKs_Calculator [58].

\section{Promoter analysis of PyyHSP70s}

The 5' upstream regions, including 2000 bp DNA sequence upstream of each PyyHSP7O gene, were subjected to the PlantCARE database (http://bioinforma tics.psb.ugent.be/webtools/plantcare/html/) for a cis-elements scan. 


\section{RNA-seq atlas analysis}

To investigate the expression patterns of PyyHSP7O genes in response to dehydration/rehydration treatments, the related RNA-sequencing (seq) data of Py. yezoensis were downloaded from NCBI under accession number PRJNA401507 [21]. The RNA-seq data of Py.haitanensis in dehydration/rehydration treatments were used to obtain expression patterns of PyhHSP7O genes [20]. Expression heatmaps were constructed using R software and based on the FPKM values of gene expression in different treatments.

\section{RNA isolation and qRT-PCR analysis}

By weighing the fresh weight and the dry weight of thalli, the absolute water content (AWC) of the thallus was calculated according to the methods described by Kim et al. (2009) [59]. Thalli produced under normal growth condition were harvested as the control group (AWC100). Before dehydration, the surface water of the thalli was removed by paper towels, and then the selected thalli were naturally dehydrated under $50 \mu \mathrm{mol}$ photons $\mathrm{m}^{-2} \cdot \mathrm{s}^{-1}$ at $8 \pm 1{ }^{\circ} \mathrm{C}$. The thalli samples were collected until the total water content decreased by $30 \%$ (AWC70), $50 \%$ (AWC50), and $80 \%$ (AWC20). After losing 80\% water content, the samples were recovered in normal seawater for 30 min (AWC20_REH) [20, 21]. Three biological replicates were performed for each treatment. Samples were harvested and placed in liquid nitrogen before processing for gene expression analysis. Total RNA was extracted using the RNeasy Plant Mini Kit (OMEGA) according to the manufacturer's instructions. Next, $1 \mu \mathrm{g}$ total RNA was used to synthesize the first-strand cDNA using a HiScript ${ }^{\circledR}$ III RT SuperMix for qPCR (+ gDNA wiper) Kit (Vazyme Biotech). The qRT-PCR analysis was performed as described previously [60]. The expression levels of the ubiquitin-conjugating enzyme (UBC) and cystathionine gamma-synthase 1 (CGS1) genes were used as reference [61]. and the $2^{-\triangle \triangle \mathrm{Ct}}$ method was used to calculate relative gene expression values. The sequences of the primers used are listed in Supplementary Table S5.

\section{Subcellular localization analysis of PyyHsp70s}

To validate the prediction of subcellular localization, transient expression analyses were performed using a protoplast system based on the pBWA(V)HS(PyyHSP70-1/ PyyHSP70-3)-GLosgfp vector. For two representative PyyHSP70 genes, the full-length CDS without the stop codon was cloned into the $\mathrm{pBWA}(\mathrm{V}) \mathrm{HS}$ vector. Each CDS was fused in-frame to the N-terminus of the green fluorescent protein (GFP) coding sequence under the control of the CaMV $35 \mathrm{~S}$ promoter. The primers used for PCR amplification of the full-length HSP7O
CDS are listed in Table S6. The vector with only GFP gene expressed was used as a control. The protoplasts used for transient expression analysis were extracted from Arabidopsis leaves and transformed by the polyethylene glycol (PEG) method [62]. Briefly, the Arabidopsis leaves was put into enzyme solution $(1.5 \%(\mathrm{w} / \mathrm{v})$ cellulose R10, 0.75\% (w/v) macerozyme R10, 0.6 M mannitol, $10 \mathrm{mM}$ MES, pH5.8) at $24{ }^{\circ} \mathrm{C}$ for $4 \mathrm{~h}$ with gentle shaking in the dark. After filtering through nylon mesh and washing two times with W5 solution (154 mM sodium chloride, $125 \mathrm{mM}$ calcium chloride $\left(\mathrm{CaCl}_{2}\right), 5 \mathrm{mM}$ glucose, $2 \mathrm{mM} \mathrm{KH_{2 }} \mathrm{PO}_{4}, 2 \mathrm{mM}$ MES, pH 5.7), protoplasts were resuspended in MMG solution (0.4 M mannitol, $15 \mathrm{mM}$ magnesium chloride, $4 \mathrm{mM}$ MES, $\mathrm{pH}$ 5.7) at a cell concentration of $2 \times 10^{5} \mathrm{~mL}^{-1}$. Then, $10 \mu \mathrm{g}$ of each plasmid sample was mixed with $100 \mu \mathrm{L}$ protoplasts, followed by addition of $120 \mu \mathrm{L}$ of freshly prepared PEG solution (40\% (w/v) PEG4000, 0.6 M mannitol, and $100 \mathrm{mM} \mathrm{CaCl}{ }_{2}$ ). The mixture was incubated at room temperature for $30 \mathrm{~min}$ in the dark, and then diluted gently with $1 \mathrm{~mL} \mathrm{~W} 5$ solution. After centrifugation at $300 \mathrm{rpm}$ for $3 \mathrm{~min}$, protoplasts were resuspended in $1 \mathrm{~mL}$ of W5 solution before incubating at $25{ }^{\circ} \mathrm{C}$ for $16 \mathrm{~h}$ and then observed using a Nikon Eclipse 80i fluorescence microscope. Respective excitation and emission wavelengths were $488 \mathrm{~nm}$ and $510 \mathrm{~nm}$ for the GFP signal, and $640 \mathrm{~nm}$ and $675 \mathrm{~nm}$ for the Chl signal.

\section{Abbreviations}

PyyHSP70: Pyropia yezoensis HSP70; CDS: Coding sequence; HMM: Hidden markov model; AWC: Absolute water content; REH: Rehydration; qRT-PCR: Quantitative real-time polymerase chain reaction.

\section{Supplementary Information}

The online version contains supplementary material available at https://doi. org/10.1186/s12870-021-03213-0.

Additional file 1: Table S1. The corresponding amino acid number of the domains in HSP70 proteins.

Additional file 2: Table S2. Motifs of HSP70 Proteins.

Additional file 3: Table S3. The gene IDs of HSP7Os.

Additional file 4: Table S4. Ka, Ks, and Ka/Ks values for duplicated ortholog pairs in Py. yezoensis and Py. haitanensis.

Additional file 5: Table S5. Gene primers designed for qRT-PCR. Additional file 6: Table S6. Primers designed for subcellular localization analysis.

Additional file 7: Figure S1. Multiple sequence alignment all 15 PyyHSP70 proteins and the ECDNAK protein. Green box: ATPase domain; orange box: interdomain hinge; blue box: peptide-binding domain; red box: C-terminal sub-domain.

Additional file 8: Figure S2. Heatmap of the expression patterns of PyhHSP70 genes under dehydration and rehydration treatments: absolute water content 100\% (AWC100, control), absolute water content 70\% (AWC70), absolute water content 20\% (AWC20), rehydrated 30 min after 20\% of water loss (AWC20_30min). The color bar represents $\log _{2}$ 
expression levels (FPKM). The tree (left) represents clustering result of PyhHSP70s' expression patterns.

\section{Acknowledgements}

We are very grateful to Professor Mao for his guidance. We thank the Key Laboratory of Marine Genetics and Breeding (OUC) and lab members for their assistance in this study.

\section{Authors' contributions}

$X Z Y$ arranged and designed the study, analyzed the data, performed the experiments and wrote the manuscript. TG completed the visualization of the data. ZLM, XHT and YXM supervised the research. All authors have read and approved the manuscript.

\section{Funding}

The authors thank for the grants supporting from the National Natural Science Foundation of China (Grant No.32060829), the National Key R\&D Program of China (2018YFD0900106), the Marine S\&T Fund of Shandong Province for Pilot National Laboratory for Marine Science and Technology (Qingdao) (No. 2018SDKJ0302-4), the Fundamental Research Funds for the Central Universities (202064006), the MOA Modern Agricultural Talents Support Project, and National Infrastructure of Fishery Germplasm Resources (2018DKA30470).

\section{Availability of data and materials}

RNA-seq data of Py. yezoensis in dehydration/rehydration treatments are available in NCBI under accession number PRJNA401507. RNA-seq data of Py. haitanensis in dehydration/rehydration treatments are available in NCBI under accession number PRJNA282903. All other datasets generated in this study are included as supplementary information of this article.

\section{Declarations}

\section{Ethics approval and consent to participate}

Not applicable.

\section{Consent for publication}

Not applicable.

\section{Competing interests}

The authors declare that they have no competing interests.

\section{Author details}

'Key Laboratory of Marine Genetics and Breeding (Ministry of Education), Ocean University of China, Qingdao 266003, China. ${ }^{2}$ College of Marine Life Sciences, Ocean University of China, 5 Yushan Road, Qingdao 266003, China. ${ }^{3}$ Key Laboratory of Utilization and Conservation of Tropical Marine Bioresource (Hainan Tropical Ocean University), Ministry of Education, Sanya 572022, China. ${ }^{4}$ Laboratory for Marine Biology and Biotechnology, Qingdao National Laboratory for Marine Science and Technology, Qingdao 266237, China.

Received: 17 March 2021 Accepted: 14 September 2021

Published online: 24 September 2021

\section{References}

1. Wang W, Vinocur B, Shoseyov O, Altman A. Role of plant heat-shock proteins and molecular chaperones in the abiotic stress response. Trends Plant Sci. 2004;9(5):244-52.

2. Sung D-Y, Kaplan F, Guy CL. Plant Hsp70 molecular chaperones: protein structure, gene family, expression and function. Physiol Plant. 2001;113(4):443-51.

3. Al-Whaibi MH. Plant heat-shock proteins: a mini review. J King Saud Univ Sci. 2011;23(2):139-50.

4. Dragovic Z, Broadley SA, Shomura Y, Bracher A, Hartl FU. Molecular chaperones of the Hsp110 family act as nucleotide exchange factors of Hsp70s. EMBO J. 2006;25(11):2519-28.
5. Sung DY, Vierling E, Guy CL. Comprehensive expression profile analysis of the Arabidopsis Hsp70 gene family. Plant Physiol. 2001;126(2):789-800.

6. Cho EK, Choi YJ. A nuclear-localized HSP70 confers thermoprotective activity and drought-stress tolerance on plants. Biotech Lett. 2009;31(4):597-606

7. Jungkunz I, Link K, Vogel F, Voll LM, Sonnewald S, Sonnewald U. AtHsp7015-deficient Arabidopsis plants are characterized by reduced growth, a constitutive cytosolic protein response and enhanced resistance to TuMV. Plant J. 2011;66(6):983-95.

8. Jung KH, Gho HJ, Nguyen MX, Kim SR, An G. Genome-wide expression analysis of HSP7O family genes in rice and identification of a cytosolic HSP70 gene highly induced under heat stress. Funct Integr Genomics. 2013;13(3):391-402

9. Guo M, Liu JH, Ma X, Zhai YF, Gong ZH, Lu MH. Genome-wide analysis of the Hsp70 family genes in pepper (Capsicum annuum L.) and functional identification of $\mathrm{CaHsp70-2} \mathrm{involvement} \mathrm{in} \mathrm{heat} \mathrm{stress.} \mathrm{Plant} \mathrm{Science.}$ 2016;252:246-56.

10. Bloch MA, Johnson KA. Identification of a molecular chaperone in the eukaryotic flagellum and its localization to the site of microtubule assembly. J Cell Sci. 1995;108(Pt 11):3541-5.

11. Silflow CD, Sun X, Haas NA, Foley JW, Lefebvre PA. The Hsp70 and Hsp40 chaperones influence microtubule stability in Chlamydomonas. Genetics. 2011;189(4):1249-60

12. Cho EK, Hong CB. Over-expression of tobacco NtHSP70-1 contributes to drought-stress tolerance in plants. Plant Cell Rep. 2006;25(4):349-58.

13. Su P-H, Li H-M. Arabidopsis stromal 70-kD heat shock proteins are essential for plant development and important for thermotolerance of germinating seeds. Plant Physiol. 2008;146(3):1231-41.

14. Alvim FC, Carolino SM, Cascardo JC, Nunes CC, Martinez CA, Otoni WC, Fontes EP. Enhanced accumulation of BiP in transgenic plants confers tolerance to water stress. Plant Physiol. 2001;126(3):1042-54.

15. Park C-J, Bart R, Chern M, Canlas PE, Bai W, Ronald PC. Overexpression of the endoplasmic reticulum chaperone $\mathrm{BiP} 3$ regulates $\mathrm{XA21}$-mediated innate immunity in rice. PLoS ONE. 2010;5(2):e9262-e9262.

16. Qi Y, Wang H, Zou Y, Liu C, Liu Y, Wang Y, Zhang W. Over-expression of mitochondrial heat shock protein 70 suppresses programmed cell death in rice. FEBS Lett. 2011;585(1):231-9.

17. Wang D, Yu X, Xu K, Bi G, Cao M, Zelzion E, Fu C, Sun P, Liu Y, Kong F, et al. Pyropia yezoensis genome reveals diverse mechanisms of carbon acquisition in the intertidal environment. Nat Commun. 2020;1 1(1):4028-4028.

18. Blouin NA, Brodie JA, Grossman AC, Xu P, Brawley SH. Porphyra: a marine crop shaped by stress. Trends Plant Sci. 2011;16(1):29-37.

19. Sun P, Mao Y, Li G, Cao M, Kong F, Wang L, Bi G. Comparative transcriptome profiling of Pyropia yezoensis (Ueda) M.S. Hwang \& H.G. Choi in response to temperature stresses. BMC Genomics. 2015;16(1):463.

20. Wang L, Mao Y, Kong F, Cao M, Sun P. Genome-wide expression profiles of Pyropia haitanensis in response to osmotic stress by using deep sequencing technology. BMC Genomics. 2015;16:1012.

21. Sun $\mathrm{P}$, Tang $X, B i \mathrm{G}, \mathrm{Xu}$ K, Kong F, Mao Y. Gene expression profiles of Pyropia yezoensis in response to dehydration and rehydration stresses. Mar Genomics. 2019;43:43-9.

22 Mittal D, Enoki Y, Lavania D, Singh A, Sakurai H, Grover A. Binding affinities and interactions among different heat shock element types and heat shock factors in rice (Oryza sativa L.). FEBS J. 2011;278(17):3076-85.

23. Davison IR, Pearson GA. Stress tolerance in intertidal seaweeds. J Phycol. 1996;32(2):197-211.

24. Alpert P. The limits and frontiers of desiccation-tolerant life. Integr Comp Biol. 2005;45(5):685-95.

25. Boorstein WR, Ziegelhoffer T, Craig EA. Molecular evolution of the HSP70 multigene family. J Mol Evol. 1994;38(1):1-17.

26. Tang L, Qiu L, Liu C, Du G, Mo Z, Tang X, Mao Y. Transcriptomic insights into innate immunity responding to red rot disease in red alga Pyropia yezoensis. Int J Mol Sci. 2019;20(23):5970.

27 Nagaraju M, Reddy PS, Kumar SA, Kumar A, Rajasheker G, Rao DM, KaviKishor PB. Genome-wide identification and transcriptional profiling of small heat shock protein gene family under diverse abiotic stress conditions in Sorghum bicolor (L.). Int J Biol Macromol. 2020;142:822-34.

28. Muthusamy SK, Dalal M, Chinnusamy V, Bansal KC. Genome-wide identification and analysis of biotic and abiotic stress regulation of small heat shock protein (HSP20) family genes in bread wheat. J Plant Physiol. 2017;211:100-13. 
29. Sarkar NK, Kundnani P, Grover A. Functional analysis of Hsp70 superfamily proteins of rice (Oryza sativa). Cell Stress Chaperones. 2013;18(4):427-37.

30. Fujita Y, Fujita M, Satoh R, Maruyama K, Parvez MM, Seki M, Hiratsu K, Ohme-Takagi M, Shinozaki K, Yamaguchi-Shinozaki K. AREB1 is a transcription activator of novel ABRE-dependent ABA signaling that enhances drought stress tolerance in Arabidopsis. Plant Cell. 2005;17(12):3470-88.

31. Yoshida T, Fujita Y, Sayama H, Kidokoro S, Maruyama K, Mizoi J, Shinozaki K, Yamaguchi-Shinozaki K. AREB1, AREB2, and ABF3 are master transcription factors that cooperatively regulate $A B R E-$ dependent $A B A$ signaling involved in drought stress tolerance and require ABA for full activation. Plant J. 2010;61(4):672-85.

32. Yamaguchi-Shinozaki K, Shinozaki K. A novel cis-acting element in an Arabidopsis gene is involved in responsiveness to drought, low-temperature, or high-salt stress. Plant Cell. 1994;6(2):251-64.

33. Nakashima K, Shinwari ZK, Sakuma Y, Seki M, Miura S, Shinozaki K, Yamaguchi-Shinozaki K. Organization and expression of two Arabidopsis DREB2 genes encoding DRE-binding proteins involved in dehydration- and high-salinity-responsive gene expression. Plant Mol Biol. 2000;42(4):657-65.

34. Liu S, Hu Z-M, Zhang Q, Yang X, Critchley AT, Duan D. PI signal transduction and ubiquitination respond to dehydration stress in the red seaweed Gloiopeltis furcata under successive tidal cycles. BMC Plant Biol. 2019;19(1):1-14

35. Kumar M, Kumari P, Reddy CRK, Jha B. Salinity and desiccation induced oxidative stress acclimation in seaweeds. Adv Bot Res. 2014;71(4):91-123.

36. Camila F, López-Cristoffanini C, Meynard A, Lovazzano C, Castaeda F. Expression profile of desiccation tolerance factors in intertidal seaweed species during the tidal cycle. Planta. 2017;245(6):1149-64.

37. López-Cristoffanini C, Tellier F, Otaíza R, Correa JA, Contreras-Porcia L. Tolerance to air exposure: a feature driving the latitudinal distribution of two sibling kelp species. Bot Mar. 2013;56(5-6):431-40.

38. Contreras-Porcia L, Lopez-Cristoffanini C, Lovazzano C, Flores-Molina MR, Reinhardt R. Differential gene expression in Pyropia columbina (Bangiales, Rhodophyta) under natural hydration and desiccation conditions. Lat Am J Aquat Res. 2013;41(5):933-58.

39. Pearson GA, Hoarau G, Lago-Leston A, Coyer JA, Kube M, Reinhardt R, Henckel K, Serrão ET, Corre E, Olsen JL. An expressed sequence tag analysis of the intertidal brown seaweeds Fucus serratus (L.) and F. vesiculosus (L.) (Heterokontophyta, Phaeophyceae) in response to abiotic stressors. Mar Biotechnol. 2010;12(2):195-213.

40. Fu W, Yao J, Wang X, Liu F, Fu G, Duan D. Molecular cloning and expression analysis of a cytosolic Hsp70 gene from Laminaria japonica (Laminariaceae, Phaeophyta). Mar Biotechnol. 2009;11(6):738-47.

41. Ji D, Li B, Xu Y, Chen C, Xie C. Cloning and quantitative analysis of five heat shock protein 70 genes from Pyropia haitanensis. J Appl Phycol. 2015:27(1):499-509.

42. Torres MA, Barros MP, Campos SC, Pinto E, Rajamani S, Sayre RT, Colepicolo P. Biochemical biomarkers in algae and marine pollution: a review. Ecotoxicology Environmental Safety. 2008;71(1):1-15.

43. Zhou X, Li X, Wang P, Yan B, Yi L, Teng Y. Expression profiles of HSP70 gene in gametophytic blade of laver Porphyra yezoensis Ueda by high temperature stress. Fish Sci. 2011;30:233-7.

44. Tang T, Yu A, Li P, Yang H, Liu G, Liu L. Sequence analysis of the Hsp70 family in moss and evaluation of their functions in abiotic stress responses. Sci Rep. 2016:6(1):33650.

45. Letunic I, Copley RR, Schmidt S, Ciccarelli FD, Doerks T, Schultz J, Ponting CP, Bork P. SMART 4.0: towards genomic data integration. Nucleic Acids Res. 2004;32(suppl_1):D142-4.

46. Collén J, Porcel B, Carré W, Ball SG, Chaparro C, Tonon T, Barbeyron T, Michel G, Noel B, Valentin K, et al. Genome structure and metabolic features in the red seaweed Chondrus crispus shed light on evolution of the Archaeplastida. Proc Natl Acad Sci USA. 2013;110(13):5247-52.

47. Schönknecht G, Chen W, Ternes C, Barbier G, Shrestha R, Stanke M, Bräutigam A, Baker B, Banfield J, Garavito R, et al. Gene transfer from bacteria and archaea facilitated evolution of an extremophilic eukaryote. Science. 2013;339(6124):1207-10.

48. Brawley SH, Blouin NA, Ficko-Blean E, Wheeler GL, Lohr M, Goodson HV, Jenkins JW, Blaby-Haas CE, Helliwell KE, Chan CX, et al. Insights into the red algae and eukaryotic evolution from the genome of Porphyra umbilicalis (Bangiophyceae, Rhodophyta). Proc Natl Acad Sci. 2017;114(31):E6361-70.

49. Cao M, Xu K, Yu X, Bi G, Liu Y, Kong F, Sun P, Tang X, Du G, Ge Y, et al. A chromosome-level genome assembly of Pyropia haitanensis (Bangiales, Rhodophyta). Mol Ecol Resour. 2020;20(1):216-27.

50. Gasteiger E, Gattiker A, Hoogland C, Ivanyi I, Appel RD, Bairoch A. ExPASy: the proteomics server for in-depth protein knowledge and analysis. Nucleic Acids Res. 2003;31(13):3784-8.

51. Bailey TL, Williams N, Misleh C, Li WW. MEME: discovering and analyzing DNA and protein sequence motifs. Nucleic Acids Res. 2006:34(suppl_2):W369-73.

52. Lin BL, Wang JS, Liu HC, Chen RW, Meyer Y, Barakat A, Delseny M. Genomic analysis of the Hsp70 superfamily in Arabidopsis thaliana. Cell Stress Chaperones. 2001;6(3):201-8.

53. Lamesch P, Berardini TZ, Li D, Swarbreck D, Wilks C, Sasidharan R, Muller R, Dreher K, Alexander DL, Garcia-Hernandez M, et al. The Arabidopsis Information Resource (TAIR): improved gene annotation and new tools. Nucleic Acids Res. 2011;40(D1):D1202-10.

54 Kumar S, Stecher G, Tamura K. MEGA7: molecular evolutionary genetics analysis version 7.0 for bigger datasets. Molecular Biology and Evolution. 2016;33(7):1870-4.

55. Larkin MA, Blackshields G, Brown NP, Chenna R, McGettigan PA, McWilliam H, Valentin F, Wallace IM, Wilm A, Lopez R, et al. Clustal W and Clustal $X$ version 2.0. Bioinformatics. 2007;23(21):2947-8.

56. Wang Y, Tang H, DeBarry JD, Tan X, Li J, Wang X, Lee T-H, Jin H, Marler B, Guo H. MCScanX: a toolkit for detection and evolutionary analysis of gene synteny and collinearity. Nucleic Acids Res. 2012;40(7):e49-e49.

57 Vatansever R, Koc I, Ozyigit II, Sen U, Uras ME, Anjum NA, Pereira E, Filiz E. Genome-wide identification and expression analysis of sulfate transporter (SULTR) genes in potato (Solanum tuberosum L.). Planta. 2016;244(6):1167-83.

58. Zhang Z, Li J, Zhao X-Q, Wang J, Wong GK-S, Yu J. KaKs_Calculator: calculating $\mathrm{Ka}$ and $\mathrm{Ks}$ through model selection and model averaging. Genomics Proteomics Bioinformatics. 2006;4(4):259-63.

59. Kim JK, Kraemer GP, Yarish C. Research note: comparison of growth and nitrate uptake by New England Porphyra species from different tida elevations in relation to desiccation. Phycol Res. 2009;57(2):152-7.

60. Yu X, Wang L, Xu K, Kong F, Wang D, Tang X, Sun B, Mao Y. Fine mapping to identify the functional genetic locus for red coloration in Pyropia yezoensis thallus. Front Plant Sci. 2020;11:867.

61. Gao D, Kong F, Sun P, Bi G, Mao Y. Transcriptome-wide identification of optimal reference genes for expression analysis of Pyropia yezoensis responses to abiotic stress. BMC Genomics. 2018;19(1):251.

62. Yoo S-D, Cho Y-H, Sheen J. Arabidopsis mesophyll protoplasts: a versatile cell system for transient gene expression analysis. Nat Protoc. 2007:2(7):1565-72.

\section{Publisher's Note}

Springer Nature remains neutral with regard to jurisdictional claims in published maps and institutional affiliations. 